\title{
Visão do paciente infantil perante atendimento odontológico em clínica universitária
}

\author{
The pediatric patient's view of dental care in a university clinic
}

\begin{abstract}
Elisabete Rabaldo Bottan Professora do Grupo de Pesquisa Atenção à Saúde Individual e Coletiva em Odontologia, Curso de Odontologia da Universidade do Vale do Itajaí (Santa Catarina, Brasil).
\end{abstract}

Fernanda AmÁBILE da SiLva

Bolsista de Iniciação Científica, curso de Odontologia da Universidade do Vale do Itajaí (Santa Catarina, Brasil).

Rodrigo Xavier Matos

Bolsista de Iniciação Científica, curso de Odontologia da Universidade do Vale do Itajaí (Santa Catarina, Brasil).

Eliane Garcia da Silveira Mestre em Odontopediatria, Curso de Odontologia da Universidade do Vale do Itajaí (Santa Catarina, Brasil).

Beatriz Helena Eger Schmitt Mestre em Odontopediatria, Curso de Odontologia da Universidade do Vale do Itajaí (Santa Catarina, Brasil).

Financiamento: Programa de Iniciação Científica da Universidade do Vale do Itajaí (Edital 04/2011 - Processo 2082).

\begin{abstract}
RESUMO
O objetivo do presente estudo foi conhecer a visão de crianças sobre o atendimento odontológico, como estratégia de avaliação dos serviços prestados em uma clínica-escola. Para a coleta de dados, este estudo exploratório com abordagem qualitativa utilizou a técnica do desenho-estória com tema. A estruturação dos dados foi efetuada com base em quatro categorias emergentes. Participaram do estudo 52 crianças com idades que variaram de 6 a 12 anos. A maioria (58\%) pertencia à faixa de 8 a 9 anos de idade, 36\% eram meninas e $64 \%$, meninos. Foram consideradas 168 evocações. A categoria "Ambiente Odontológico" foi a mais frequente, com $34,5 \%$, indicando uma familiaridade positiva dos pesquisados com o consultório odontológico. A categoria "Imagem do cirurgião-dentista" obteve 31,6\% das evocações, com ênfase na relação humanizada. A frequência de cada uma das outras duas categorias foi 19,6\%, para "Comportamento durante o Atendimento Odontológico", e 14,3\% para "Atenção Odontológica Prestada"; em ambas predominou a conotação positiva. O atendimento odontopediátrico desenvolvido nesta universidade, na visão dos pesquisados, configura-se como uma situação agradável. O processo avaliativo, pela técnica do desenho-estória, foi uma excelente alternativa metodológica para se obter informações sobre os serviços prestados por esta instituição.
\end{abstract}

Palavras-chave: ASSISTÊNCIA ODONTOLÓGICA PARA CRIANÇAS; AVALIAÇÃO EM SAÚDE; CUIDADOS DE SAÚDE. 


\begin{abstract}
The aim of this study was to understand children's view of dental care, to assess the services provided by a school clinic. For data collection, this exploratory qualitative study used the story-drawing technique with a theme. The data structuring was carried out based on four emerging categories. The study included 52 children with ages ranging from 6 to 12 years. The majority (58\%) was 8 to 9 years old, 36\% were girls and $64 \%$ were boys. One hundred and sixty eight evocations were considered. The "Dental Environment" category was the most frequent, with $34.5 \%$, indicating the respondents' positive familiarity with the dental office. The "Dentist's image" category got $31.6 \%$, with an emphasis on the humanized relationship. The frequency of each of the other two categories was 19.6\% for "Behavior during Dental Treatment", and 14.3\% for "Dental Care Provided"; in both of them the positive connotation predominated. The pediatric dental care provided by this university, in the view of respondents, appears as a pleasant situation. The evaluation process, through the story-drawing technique, was an excellent alternative methodology to gather information about the services provided by this institution.
\end{abstract}

Keywords: DENTAL CARE FOR CHILDREN; HEALTH ASSESSMENT, HEALTH CARE.

\section{INTRODUÇÃO}

A avaliação de ações em saúde, indiscutivelmente, é uma atividade que vem crescendo. Nas últimas décadas, no Brasil, diversas iniciativas para avaliação de serviços de saúde têm sido desenvolvidas, com abordagens definidas entre pesquisas acadêmicas e pesquisas avaliativas, cujos resultados vêm sendo incorporados pelo MinistériodaSaúde, para subsidiara elaboração de políticas e programas setoriais. 1,2,3,4

Esta condição tem gerado uma diversificação de estratégias metodológicas que podem ser agrupadas em dois tipos: avaliação subjetiva e pesquisa avaliativa. A avaliação subjetiva emprega o processo geral de julgamento de valor e utiliza, basicamente, a opinião de usuários e de especialistas. A pesquisa avaliativa emprega, especificamente, o método científico com o propósito de efetuar uma análise pontual do objeto de interesse..$^{1,3,4}$

O envolvimento dos usuários em processos avaliativos contribui com a melhoria da qualidade dos serviços prestados, pois sua perspectiva fornece informações essenciais. A avaliação compartilhada é um instrumento dinâmico de transformação, visto que a avaliação em si constitui-se em um processo capaz de abrigar as vozes dos diferentes atores sociais. ${ }^{1,2,5}$

Pesquisas com abordagem qualitativa que investiguem o significado do atendimento odontológico em diferentes grupos sociais, em diferentes faixas etárias, são fundamentais para a área odontológica. $6,7,8$

Em se tratando do atendimento prestado à clientela infantil, o processo avaliativo geralmente é realizado pela percepção dos pais ou responsáveis. Todavia, o relato dos pais/responsáveis pode não refletir de forma fidedigna todos os aspectos que traduzem a visão da criança quanto ao atendimento que está recebendo no consultório odontológico. ${ }^{9}$

A visão da criança sobre a experiência odontológica vivenciada é de suma importância para a compreensão da prática odontológica desenvolvida no âmbito das diferentes instituições que oferecem este tipo de serviço. Em consonância com este pensamento, encontra-se esta investigação, cujo objetivo foi conhecer a visão de pacientes infantis sobre o atendimento odontológico como uma estratégia de avaliação dos serviços prestados pela Clínica de Odontopediatria do curso de Odontologia da Universidade do Vale do Itajaí (Univali, SC).

\section{Metodologia}

A pesquisa caracteriza-se como uma investigação exploratória, com abordagem qualitativa. Os sujeitos do estudo foram crianças de 6 a 12 anos de idade, em atendimento na Clínica de Odontopediatria do curso de Odontologia da Univali no período de outubro a novembro de 2011 e de março a maio de 2012. De acordo 
com os registros, esta clínica possui uma média mensal de 120 atendimentos.

O projeto de pesquisa foi aprovado pela Comissão de Ética em Pesquisa da Univali sob o $\mathrm{n}^{\circ} 190$ e todos os procedimentos foram conduzidos em conformidade com os preceitos éticos, segundo a Resolução 196/96.

O número de crianças que integraram a pesquisa foi delimitado pela técnica da saturação dos dados. ${ }^{10}$ Para a obtenção dos dados, foi utilizada a técnica do desenho-estória com tema. Esta é uma técnica projetiva constituída pela associação entre um processo expressivo-motor, que adota o desenho livre, e um procedimento perceptivo-dinâmico, que utiliza a verbalização temática. ${ }^{6,8,10}$

A partir do desenho-estória, obteve-se o corpus, que é o conjunto de dados estruturado a partir da transcrição das falas e da análise dos desenhos elaborados pelas crianças. Considerando-se a abordagem qualitativa do estudo, optou-se pela construção de um corpus, em substituição a uma amostragem representativa.

A coleta de dados ocorreu com uma frequência semanal, seguindo-se o calendário acadêmico, no período proposto (outubro a novembro de 2011 e de março a maio de 2012).

Os sujeitos eram abordados ao chegarem à sala de espera da clínica, quando os pesquisadores apresentavam-se e explicavam às crianças e aos pais/responsáveis os procedimentos e os objetivos da pesquisa. Para os que aceitavam participar da pesquisa, por livre e espontânea vontade, era solicitada a assinatura do Termo de Consentimento Livre e Esclarecido pelo responsável.

Os critérios para inclusão dos sujeitos no estudo foram: a) ter efetivado pelo menos três sessões de atendimento na clínica-escola; b) aceitar participar da pesquisa; c) não ser portador de deficiência mental.

Foi solicitado que a criança elaborasse um desenho sobre $\mathrm{o}$ atendimento odontológico recebido na clínica da Univali. Para a elaboração do desenho foram disponibilizados folha de papel sulfite branco, lápis de cor, lápis de cera, lápis preto, borracha e apontador. Posteriormente, cada criança descrevia seu desenho individualmente, sendo esta conversa gravada.

A análise dos dados foi efetuada com base na técnica de análise temática. ${ }^{11}$ Inicialmente, foi realizada a leitura flutuante, que consiste na tomada de contato inicial com o material produzido (desenhos e transcrição das falas) de modo assistemático. Em seguida, foi realizada a observação sistemática dos desenhos, com agrupamento por semelhanças, e a leitura sistemática dos textos obtidos com a transcrição das falas, complementando a interpretação dos desenhos.

Foram incluídos no estudo apenas aqueles desenhos que atenderam aos seguintes quesitos: enfoque do tema proposto; estar concluído; ser claro para interpretação, de modo isolado ou com ajuda da descrição oral efetuada pela criança.

Em cada categoria, os indicadores foram dicotômicos, para evidenciar uma conotação positiva ou negativa do item avaliado, conforme Quadro 1.

A interpretação dos desenhos foi realizada por três pesquisadores, que utilizaram o roteiro descritivo das categorias. Para a determinação donível deconcordância entre os pesquisadores foi previamente desenvolvido um estudo piloto, cujos dados não foram incluídos na pesquisa. As manifestações foram tabuladas segundo as categorias, sendo calculada a frequência relativa de cada uma.

\section{Resultados}

Participaram do estudo 52 crianças com idades que variavam de 6 a 12 anos. A maioria (58\%) estava na faixa dos 8 a 9 anos de idade. O gênero masculino foi o mais frequente (64\%).

Por meio da técnica do desenho-estória foram obtidas 168 evocações, distribuídas nas 


\section{Quadro 1 - Categorias e subcategorias preestabelecidas adotadas no estudo}

\begin{tabular}{|c|l|}
\hline CATEGORIAS & \multicolumn{1}{c|}{ INDICADORES } \\
\hline Ambiente do consultório & $\begin{array}{l}\text { - Conotação positiva: familiarização com objetos, materiais e instrumentais do } \\
\text { consultório odontológico. } \\
\text { - Conotação negativa: ausência de objetos, materiais e instrumentais do consultó- } \\
\text { rio odontológico ou indicação com ausência de detalhes ou caracterização destes } \\
\text { objetos como aterrorizadores. }\end{array}$ \\
\hline Atenção odontológica prestada & $\begin{array}{l}\text { - Conotação positiva: evidências da valorização da abordagem clínica associada } \\
\text { à abordagem educativo-preventiva (cuidados de higiene bucal, consulta de rotina, } \\
\text { repasse de orientações). } \\
\text { - Conotação negativa: evidências da valorização apenas dos procedimentos clí- } \\
\text { nicos. }\end{array}$ \\
\hline Imagem quanto ao CD* & $\begin{array}{l}\text { - Conotação positiva: evidências de uma relação humanizada entre paciente e } \\
\text { CD (diálogo, companheirismo, empatia, afetividade). } \\
\text { - Conotação negativa: evidências de antipatia, de valorização do procedimento } \\
\text { em detrimento da relação profissional-paciente. }\end{array}$ \\
\hline Comportamento durante o atendimento odon- & $\begin{array}{l}\text { - Conotação positiva: manifestações de comportamento participativo, de alegria, } \\
- \text { Conotação negativa: manifestações de medo, ansiedade, nervosismo, esquiva. }\end{array}$ \\
\hline
\end{tabular}

${ }^{*} \mathrm{CD}=$ Cirurgião-dentista.

quatro categorias de análise. A maioria das evocações relacionava-se à descrição do ambiente do consultório odontológico e à imagem do cirurgião dentista (CD). A frequência de cada categoria pode ser identificada na Tabela 1.

Tabela 1 - Distribuição da frequência das evocações de cada categoria

\begin{tabular}{lrc}
\hline & \multirow{2}{*}{ EVOCAÇÕES POR CATEGORIAS } & \multicolumn{2}{c}{$\mathbf{N}^{\circ}$} & $\%$ \\
\hline Ambiente do consultório & $\mathbf{5 8}$ & $\mathbf{3 4 , 5}$ \\
Conotação positiva & 53 & 31,5 \\
Conotação negativa & 05 & 3,0 \\
\hline Imagem quanto ao CD & $\mathbf{5 3}$ & $\mathbf{3 1 , 6}$ \\
Conotação positiva & 50 & 29,8 \\
Conotação negativa & 03 & 1,8 \\
\hline Comportamento no atendimento odontológico & $\mathbf{3 3}$ & $\mathbf{1 9 , 6}$ \\
Conotação positiva & 27 & 16,1 \\
Conotação negativa & 06 & 3,6 \\
\hline Atenção odontológica prestada & $\mathbf{2 4}$ & $\mathbf{1 4 , 3}$ \\
Conotação positiva & 19 & 11,3 \\
Conotação negativa & 05 & 3,0 \\
\hline
\end{tabular}


O ambiente do consultório odontológico foi descrito com riqueza de detalhes nos desenhos e falas que evidenciaram materiais, instrumentais e equipamentos, bem como a presença do acadêmico que prestava o atendimento da criança, como pode ser comprovado no desenho elaborado por uma das crianças (Figura 1).

\section{Figura 1 - Destaque às categorias "Ambiente} do Consultório" e "Imagem do CD"

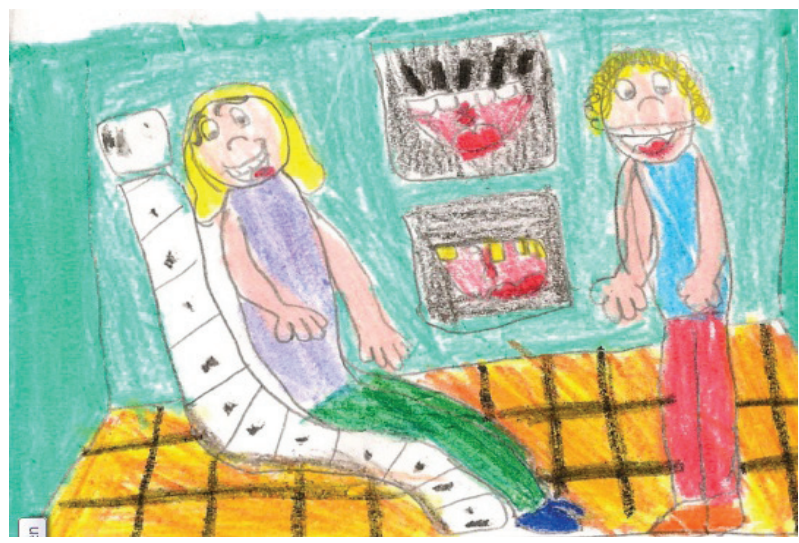

Dentreasfalassobreos desenhos produzidos foram destacadas e transcritas algumas que representam as diferentes categorias de análise. Optou-se pela apresentação literal das falas para que não se perdesse a força de expressão dos pesquisados.

\section{Categorias: Ambiente do Consultório (AC) e Comportamento no Atendimento Odontológico (CAO)}

"No consultório do dentista tem aquela luz pra ver o dente, tem as folha que eles escreve" (AC).

"Eu gosto de ir no dentista, só não gosto da aguIha" (CAO).

\section{Categorias: Ambiente do Consultório (AC), Imagem do CD (ICD) e Comportamento no Atendimento Odontológico (CAO)}

"Eu gosto do dentista daqui. Eu gosto dos dentistas que me atende" (ICD).

\begin{abstract}
"Gosto quando eles usam a vassourinha no meu dente, só não gosto da anestesia, porque dói a picada" (AC).

"[...] eles [referindo-se aos acadêmicos] são muito legal. Eles conversam; eles explicam tudo. Eles até dão escova e pasta" (ICD).
\end{abstract}

\section{Categorias: Atenção Odontológica Prestada (Educativo-Preventiva) (AOEP), Imagem do $C D$ (ICD) e Comportamento no Atendimento Odontológico (CAO)}

\begin{abstract}
"Eu desenhei assim! Pra deixar o dente limpinho tem que usar a escova, com a pasta e o fio dental, que tira essas sujeirinhas e daí o dente fica branco. Se não escova eles ficam igual aqueles ali, tudo sujo e feio. O meu dentista me ensina tudo prá fazer certinho" (AOEP).

"Esse é eu escovando os dentes. O pontinho amarelo é sujeira no meu dente. Daí tenho que escovar pra meu dente não pegar cárie. Por isso eu gosto de ir neste dentista daqui porque eles ensinam bem e eles são muito legal. Eles conversam e explicam bem. Eu nem tenho medo de ir no dentista" (AOEP; ICD; CAO).
\end{abstract}

\section{Dıscussão}

Desenhar, para a criança, é como brincar. Portanto, o desenho é uma técnica eficiente para demonstrar a visão que a criança tem sobre o atendimento que recebe em clínicas odontológicas. O desenho é elaborado a partir de interações sociais que se refletem no grafismo. ${ }^{7,12} \mathrm{O}$ ato de desenhar é uma atividade agradável e de fácil execução que favorece a evocação de significados. ${ }^{7,12-15} \mathrm{E}$ isto pode ser efetivamente observado no transcurso desta investigação. Todas as crianças, após as explicações sobre a pesquisa, prontamente aceitaram o convite e expressaram satisfação em desenhar e relatar seus desenhos.

A análise dos desenhos-estória sobre o atendimento odontológico prestado pela Clínica de Odontopediatria da Univali evidenciou aspectos muito positivos quanto às ações desenvolvidas por acadêmicos e professores. Um dos aspectos mais propícios para avaliar a 
satisfação de uma comunidade em relação ao curso de Odontologia é o atendimento prestado por suas clínicas, por ser o retrato do trabalho de uma equipe. ${ }^{1}$ Portanto, a visão do paciente infantil que integrou este estudo reflete a eficácia do trabalho realizado pelas equipes da Clínica de Odontopediatria da Univali.

A descrição dos desenhos, por meio das falas das crianças, denotou um cenário de tranquilidade e empatia. Ficou muito evidente que há uma relação de confiança, uma boa comunicação entre os acadêmicos e estas crianças. A comunicação entre o dentista e a criança, objetivada por um relacionamento amigável e amistoso durante o atendimento, é essencial para o sucesso do tratamento odontológico e, portanto, para o estabelecimento de comportamentos saudáveis. Esta condição foi percebida nos desenhos-estória realizados pelos pacientes da Clínica de Odontopediatria da Univali.

Um cenário de cordialidade, comunicabilidade, boa vontade, aliado à competência técnica, potencializa o relacionamento paciente-profissional, favorecendo e valorizando o trabalho odontológico. 8,14,15,16,17

A avaliação dos pacientes infantis pesquisados faz emergir a postura profissional que o curso de Odontologia da Univali tem assumido no decorrer da formação de seus acadêmicos. Esta postura, definida pelas Diretrizes Curriculares para o Curso de Odontologia ${ }^{18}$, destaca os seguintes aspectos do perfil do profissional: precisa ter uma visão generalista; saber comunicar-se com o paciente; ser ético e; principalmente, considerar o paciente um ser humano único e especial.

O paciente infantil que manifesta um comportamento positivo diante do atendimento odontológico é aquele que convive com condutas profissionais positivas, tais como o repasse adequado de informações e a compreensão das reações emocionais da criança, $, 13,14,16,17$ situação esta que foi muito enfocada nos desenhos-estória.

As crianças demonstraram que são orientadas quanto aos cuidados essenciais para o estabelecimento de boas condições de saúde bucal. Estes procedimentos contribuem para a conscientização quanto aos comportamentos adotados pelos sujeitos em relação à saúde, permitindo perceber a relação saúde-doença na perspectiva da melhoria da qualidade de vida mediante a substituição de estilos de vida. . $^{6}$

O desenvolvimento de aptidões pessoais, por meio da educação do paciente, é uma importante estratégia de atenção primária à saúde, que compreende esforços capazes de promover mudanças de comportamento e estabelecer hábitos que proporcionem prevenção/controle das doenças.

A ênfase na imagem humanizada do dentista, bem como os comportamentos altamente positivos diante do atendimento odontológico expressos pelos pesquisados, permite-nos inferir que os acadêmicos que prestam atendimento odontológico a estas crianças estão preocupados em fazer com que seus pacientes compreendam a importância do autocuidado para o alcance de melhores níveis de saúde.

\section{ConsideraçõEs finaIS}

Diante das exposições das crianças por meio de seus desenhos-estórias, pode-se afirmar que, para a maioria dos sujeitos integrantes da pesquisa, o contexto da consulta odontológica revela-se uma situação agradável, caracterizada por uma prática educativo-preventiva e permeada por uma visão humanizada do profissional da odontologia.

Os resultados obtidos são condizentes com a filosofia norteadora do curso de Odontologia da Univali, que preconiza a formação de profissionais com uma conduta humanizada 
e uma visão generalista do paciente, considerando seus aspectos biopsicossociais.

$E$, finalmente, constatou-se que $O$ processo avaliativo, por meio da técnica do desenho-estória, é rico e autêntico. Portanto, a técnica pode ser considerada uma excelente alternativa metodológica quando comparada à utilização de questionários, os quais podem induzir as respostas dos pesquisados, limitando a qualidade e a profundidade do processo de avaliação.

\section{Agradecimentos}

Ao Programa de Iniciação Científica ProBIC, da Pró-Reitoria de Pesquisa, PósGraduação, Extensão e Cultura da Univali, pelo financiamento da pesquisa.

\section{REFERÊNCIAS BIBLIOGRÁFICAS}

1. Bottan ER, Sperb RAL, Telles OS, Uriarte Neto M. Avaliação de serviços odontológicos: a visão dos pacientes. Rev ABENO. 2006; 6(2): 128-33.

2. D'Avila S, Souza LMM, Cavalcanti GMS, Lucas RSCC, Granville-Garcia AF, Cavalcanti AL. Nível de satisfação e condição socioeconômica dos usuários das clínicas de odontologia da Universidade Estadual da Paraíba. Rev Bras Pesq Saúde. 2010; 12(2): 39-45.

3. Sisson MC, Oliveira MC, Conill EM, Pires D, Boing AF, Fertonani HP. Satisfação dos usuários na utilização de serviços públicos e privados de saúde em itinerários terapêuticos no sul do Brasil. Interface Comun Saúde Educ. 2011; 15(36): 123-36.

4. Soller SAL, Regis Filho GI. Uso de indicadores da qualidade para avaliação de prestadores de serviços públicos de odontologia: um estudo de caso. RAP. 2011; 45(3): 591-610.

5. Lima ACS, Cabral ED, Vasconcelos MMVB. Satisfação dos usuários assistidos nos centros de especialidades odontológicas do município de Recife, Pernambuco, Brasil. Cad Saúde Públi. 2010; 26(5): 991-1002.

6. Alves RD. O tratamento odontológico sob o olhar da criança: um estudo de representações sociais [dissertação de mestrado]. Natal:
Universidade Federal do Rio Grande do Norte, Programa de Mestrado em Odontologia; 2005.

7. Montoni KMMC, Tenório MDH, Santos LM, Santos NB. Percepção dos escolares de ensino fundamental da rede pública da cidade de Maceió-AL sobre a consulta odontológica. Rev Bras Pesqui Saúde; 2009; 11(2): 24-9.

8. Ketzer JC, Bottan ER, Araujo SM, Rocha ALH. A visão de crianças sobre o atendimento odontológico em função do tipo de instituição escolar (pública ou privada). Pesqui Bras Odontoped Clin Integr. 2012; 12(4): 541-47.

9. Massoni ACLT, Ferreira JMS, Colares V, Duarte RC. Roteiro para interpretação de desenhos: facilitando a abordagem da criança no consultório odontológico. Arq Odontol. 2008; 44(3): 31-6.

10. Bauer MW, Aarts B. A construção do corpus: um princípio para coleta de dados qualitativos. In: Bauer MW, Gaskell G. Pesquisa qualitativa com texto, imagem e som. Petrópolis: Vozes; 2002. p. 39-63.

11. Bardin E. Análise de conteúdo. Lisboa: Edições 70; 2002.

12. Menezes M, Moré CLOO, Cruz RM. O desenho como instrumento de medida de processos psicológicos em crianças hospitalizadas. Aval Psicol. 2008; 7(2): 189-98.

13. Amorim VCS, Santos MFS. Visão que a criança tem do dentista através da interpretação de desenhos. Rev ABO Nac. 2000; 7(6): 359-63.

14. Hanna LNO, Araújo RJG, Gabriel DP, Nogueira AJS. O perfil do dentista sob olhar infantil. Ezamaz. 2009; 1(1): 24-37.

15. Oliveira FCM. Compreendendo a fobia em odontopediatria por meio de intervenções com o procedimento de desenhos-estórias [dissertação de mestrado]. São Paulo: Universidade de São Paulo, Programa de Mestrado em Psicologia; 2008.

16. Bottan ER, Dall' Oglio J, Silveira EG, Marchiori SA. Cirurgião-dentista ideal: perfil definido por crianças e adolescentes. RSBO. 2009; 6(4): 381-6.

17. Macerou RTP. Representação social do cirurgião dentista e o comportamento infantil, face ao tratamento odontopediátrico [dissertação de 
mestrado]. Campo Grande: Universidade Católica Dom Bosco, Programa de Mestrado em Psicologia; 2004.

18. Brasil. Ministério da Educação. Conselho Nacional de Educação. Resolução CNE/CES $\mathrm{n}^{\circ} 3$, de 19 de fevereiro de 2002. Diário Oficial da União, Brasília, 4 de março de 2002. Seção 1, p. 10. Disponível em <http://portal.mec.gov. $\mathrm{br} / \mathrm{cne} /$ arquivos/pdf/CES032002.pdf>. Acesso em: 03 junho 2013.
Endereço para Correspondência:

Elisabete Rabaldo Bottan

Av. Atlântica, 1020, ap. 1801- Balneário Camboriú - SC

CEP: 88330-006 - Fone/Fax (47) 33417564.

E-mail: erabaldo@univali.br

Submetido em: 18-9-2013

Aceito em: 23-10-2013 